\title{
Effectiveness of alteplase in the very elderly after acute ischemic stroke
}

\author{
This article was published in the following Dove Press journal: \\ Clinical Interventions in Aging \\ 23 July 2013 \\ Number of times this article has been viewed
}

\section{Josef Yayan}

Department of Internal Medicine, University Hospital of Saarland, Homburg/Saar, Germany
Correspondence: Josef Yayan Department of Internal Medicine, University Hospital of Saarland, Kirrberger Straße, D-6642I Homburg/ Saar, Germany

Tel +49684 II62 I620

Fax +49684 II 623602

Email josef.yayan@hotmail.com

\begin{abstract}
Alteplase has traditionally been the only pharmacologic agent available for treating acute ischemic stroke worldwide, and is considered an effective and safe therapeutic drug for acute cerebral ischemia. However, the drug is usually indicated for use in patients aged $<81$ years due to insufficient literature regarding the drug's safety in older individuals. Nevertheless, the elderly can benefit from alteplase after they experience an acute ischemic stroke. Age differences have been observed in the clinical presentation of acute ischemic stroke; however, the safety and efficacy of alteplase for patients with acute ischemic stroke do not depend on age or sex. Evidence of an increasing rate of intracerebral hemorrhage among the elderly with acute ischemic stroke, following alteplase treatment, has not been reported. Severe intracranial hemorrhage is a known side effect of alteplase but is not associated with age in patients with acute ischemic stroke. Alteplase can be used safely and effectively to treat elderly patients who suffer an acute ischemic stroke, including those over the age of 80 years.
\end{abstract}

Keywords: tissue-plasminogen activator, old age, safety, tolerability, intracerebral hemorrhage

\section{Introduction}

Stroke and its consequences are leading causes of significant disability and death worldwide. Stroke can occur at any age, and advancing age is a risk factor. ${ }^{1}$ Intravenous administration of the recombinant tissue-plasminogen activator (rt-PA) is the only approved thrombolytic treatment for patients with acute ischemic stroke. ${ }^{2}$ Elderly, especially those aged $>80$ years, often present in low numbers in randomized clinical trials demonstrating the lack of data providing clear evidence of rt-PA safety in those aged above 80 years. $^{2}$ Thus, alteplase is not routinely used to treat acute ischemic stroke in those aged $>80$ years in some countries, even though these patients may benefit from this therapy. ${ }^{2}$

In recent years, emergency management and treatment protocols for stroke have been evaluated. ${ }^{2}$ Effective management is crucial and begins with the organization of the entire treatment chain, from the community and prehospital setting, through the emergency department, to a dedicated stroke unit, and on to comprehensive rehabilitation. ${ }^{2}$ If patients meet the strict eligibility criteria for treatment by presenting within the extended therapeutic time window within 4.5 hours of the onset of symptoms, intravenous administration of $0.9 \mathrm{mg} / \mathrm{kg}$ (maximum dose, $90 \mathrm{mg}$ ) of alteplase can improve the outcomes of acute ischemic stroke, despite the associated increased risk of intracranial hemorrhage. ${ }^{3}$ Further, postmarketing studies have confirmed that alteplase can be administered in various hospital settings for acute ischemic stroke. ${ }^{2}$ 
Intra-arterial revascularization should also fully restore cerebral flow by providing intra-arterial thrombolytic therapy and improving clinical outcomes. ${ }^{2}$ This procedure can be performed within the first few hours of the onset of symptoms in patients who also have occlusion of the middle cerebral artery and the basilar artery. ${ }^{2}$ However, this method requires further research because intra-arterial therapy has not been proven to improve clinical outcomes.

Although intravenous alteplase is recommended for patients aged $>18$ years who experience acute ischemic stroke, patients aged $>80$ years have often been excluded or underrepresented in randomized clinical trials of thrombolysis. Therefore, evidence of the risks and benefits of alteplase use in the very elderly remains quite limited. ${ }^{2}$ A prospective, multicenter, national, open-label randomized study was started to demonstrate the efficacy and safety of intravenous alteplase in patients aged $>80$ years and treated with intravenous rt-PA within 3 hours of an ischemic stroke. ${ }^{4}$ Patients treated with alteplase were compared with a control group who received standard treatment according to national guidelines $;{ }^{4}$ however, this Italian multicenter study is ongoing, and the results are not available.

This review evaluated the use of intravenous alteplase for treating acute ischemic stroke in the very elderly (aged $>80$ years) compared with those aged $<80$ years. An extensive search in the PubMed database was conducted for articles published from 1950 to 2012, which addressed the safe use of rt-PA in elderly stroke patients aged $>80$ years.

\section{Efficacy of alteplase in patients aged $>\mathbf{8 0}$ years Efficacy and safety}

In a separate Italian multicenter study, the use of intravenous thrombolysis in patients aged $>80$ years was evaluated and compared with that in younger individuals to determine the incidence of good functional outcomes 3 months after the stroke. Good outcomes were defined as those with modified Rankin Scale (mRS) scores of 0-2, and poor outcomes included death or dependency (mRS scores of 3-5). According to the study results, no difference in the incidence of intracerebral hemorrhage was observed between the groups. Additionally, the positive and negative outcomes of the thrombolytic treatment were not statistically different between the two cohorts after 3 months. Those aged $>80$ years had a statistically higher mortality rate than the younger group. The National Institutes of Health Stroke Scale (NIHSS) score predicted mortality and poor outcomes in the older group. However, the final outcome of this study suggested that thrombolytic therapy should also be available to elderly patients, ie, those aged $>80$ years (Table 1$){ }^{5}$

Pundik et $\mathrm{al}^{6}$ investigated the possibility of a relationship between brain hemorrhage after intravenous or intra-arterial lysis with alteplase and factors other than an age $>80$ years. The authors examined the associations between age ( $>80$ years), demographic characteristics, onset to treatment time, severity of neurologic deficits, route of administration, blood glucose levels, and mean blood pressure with symptomatic intracranial hemorrhage after treatment with alteplase. Of these factors, only hyperglycemia was associated with an increased risk of intracranial hemorrhage after alteplase treatment in this patient population. Since the rates of intracerebral hemorrhage were similar for the populations, irrespective of age, the authors concluded that the decision regarding the use of alteplase should not be based only on the patient's age. ${ }^{6}$

The authors of another study, the Canadian Alteplase for Stroke Effectiveness Study, ${ }^{7}$ arrived at the same conclusion as Pundik et al. ${ }^{6}$ Intravenous administration of alteplase was not associated with an increased risk of intracerebral hemorrhage in patients aged $>80$ years. In this prospective study, age-dependent differences were found only in the clinical symptoms and positive outcomes, defined as mRS scores of $0-1$ after 90 days, in the more elderly ( $>80$ years) study population. A multivariate logistic regression analysis showed that age $>80$ years, stroke severity, baseline Alberta Stroke Program Early Computed Tomography Scores, ${ }^{7}$ and blood glucose levels were independent predictors of outcome. Careful selection of elderly patients for thrombolysis with alteplase ensured that the risk of intracranial bleeding was not increased. ${ }^{?}$

In a previous study, ${ }^{8}$ after acute ischemic stroke, elderly patients demonstrated a higher NIHSS score compared with patients aged $<80$ years. As a result, the more elderly ( $>80$ years) patients derived less benefit from the thrombolysis treatment and had increased stroke mortality. Baseline NIHSS score and age were the sole predictors of stroke outcome. However, a threshold value was not found for age and baseline NIHSS score predicting stroke outcome. ${ }^{8}$

According to Gracia-Caldentey et al, ${ }^{9}$ female sex, hypertension, increased blood pressure at admission, cardioembolic ischemic stroke, and a higher basal NIHSS score were more common after acute ischemic stroke in elderly patients aged $>85$ years. The rate of intracerebral hemorrhage with rt-PA treatment was similar for patients older and younger than 85 years of age. However, the mortality rate within 3 months was higher in those aged $>85$ years. ${ }^{9}$ 
To determine the risks and advantages of intravenous thrombolysis in patients aged $>80$ years who have experienced an acute ischemic stroke, another study compared the outcomes and symptomatic cerebral hemorrhage rates between patients aged $<80$ years and those aged $>80$ years. The study endpoints included mortality and independence, based on a mRS score of $0-2$ at 3 months, and symptomatic intracerebral hemorrhage rates. The symptomatic intracerebral hemorrhage rates were based on definitions of a Safe Implementation of Treatment in Stroke (SITS) ${ }^{10} \geq 4$-point deterioration in the NIHSS score within 36 hours, and the number of type 2 parenchymal hemorrhages according to the National Institute of Neurological Disorders and Stroke definitions. ${ }^{10}$ The intracranial hemorrhage rates were not statistically significant after adjusting for other risk factors in those aged $>80$ years compared with the younger individuals. However, patients aged $>80$ years had a higher mortality rate and reduced independence (Table 1 ). The rates of symptomatic intracranial hemorrhage were similar in the two groups. Higher mortality and worse functional outcomes were consistent with the generally poor prognosis seen in the natural history of an older age group. ${ }^{10}$

In the past, patients aged $>80$ years were excluded from randomized controlled studies examining alteplase treatment for acute ischemic stroke. Therefore, post-treatment data for patients aged $>80$ years are limited in the medical literature. Engelter et $\mathrm{al}^{11}$ examined the available studies and published the results of a systematic literature search in 2006. The authors compared six studies investigating the outcomes and side effects of intravenous rt-PA in patients with acute ischemic stroke and compared the results for patients aged above and below 80 years. Although the mortality rate was higher in patients aged $>80$, the incidence of intracerebral hemorrhage, as a treatment complication, was not significantly different between the age groups. ${ }^{11}$

In 2004, Simon et al ${ }^{12}$ analyzed the efficacy and safety of rt-PA use in patients with acute ischemic stroke and aged $>80$ years. Although the in-hospital mortality rate, NIHSS score, and incidence of symptomatic intracerebral hemorrhage were higher in older stroke patients, the authors concluded that the more elderly $(>80)$ patients should not be excluded from treatment with rt-PA based on their age alone. ${ }^{12}$

In 2011, Sung et al ${ }^{13}$ showed that symptomatic intracerebral hemorrhage was not statistically different between those aged above and those aged below 80 years. The baseline NIHSS score and the number of patients discharged were not statistically different for the younger and older patients. Although the older patients could be safely treated with intravenous rt-PA, the authors did not support the routine use of rt-PA in these older patients. ${ }^{13}$

With advanced age, patients are at a higher risk of a poor outcome following a stroke. The outcome after acute ischemic stroke was better in patients over 80 years of age following rt-PA treatment than if they did not receive such treatment in the Mishra et al study. ${ }^{14}$ The risk of rt-PA intravenous use in the elderly is considered high, but some studies have identified the benefits of alteplase treatment in these patients. Despite the higher mortality rate in patients, the symptomatic or fatal intracerebral hemorrhage and the recanalization rates after rt-PA treatment did not differ between patients older and younger than 80 years of age. ${ }^{15}$ Higher rates of neurologic improvement and positive clinical outcome were found among acute ischemic stroke patients aged $>80$ years treated with rt-PA compared to those who did not receive thrombolytic treatment. These authors recommended the use of rt-PA to treat older patients, after individuals for whom treatment was contraindicated were excluded. ${ }^{15,16}$

Longstreth et al found that patients over 80 years old were 2.87 times more likely to experience symptomatic intracerebral hemorrhage after treatment with rt-PA than were younger patients. ${ }^{17}$ However, a different study found a beneficial outcome for alteplase treatment in the older population. ${ }^{18}$ The in-hospital mortality rate did not increase among patients aged $>80$ years and treated with rt-PA. However, advanced age ( $>80$ years) was an independent predictor of mortality and a less favorable functional outcome after an acute ischemic stroke. Although the outcomes for patients in this age group were poor, the results of a previous study showed that an equal ratio of patients in this age group demonstrated earlier neurological improvement after treatment with rt-PA compared to their younger counterparts. ${ }^{19}$ Henriksen et al ${ }^{20}$ found similar results after patients aged $>80$ years who had experienced acute ischemic stroke were treated with rt-PA. The authors found that advanced age could be an independent risk factor for intracerebral hemorrhage and mortality after alteplase treatment, but the treatment did not affect good functional outcomes. Therefore, the authors proposed alteplase therapy for patients in this age group who experience acute ischemic stroke. ${ }^{20}$

Another study demonstrated that early alteplase treatment for patients aged $>80$ years appeared to be safe and effective, with those treated early recovering rapidly, showing an improvement in their NIHSS scores in 3 days, and demonstrating continuous improvement in the Barthel index. However, several elderly patients were excluded from the 


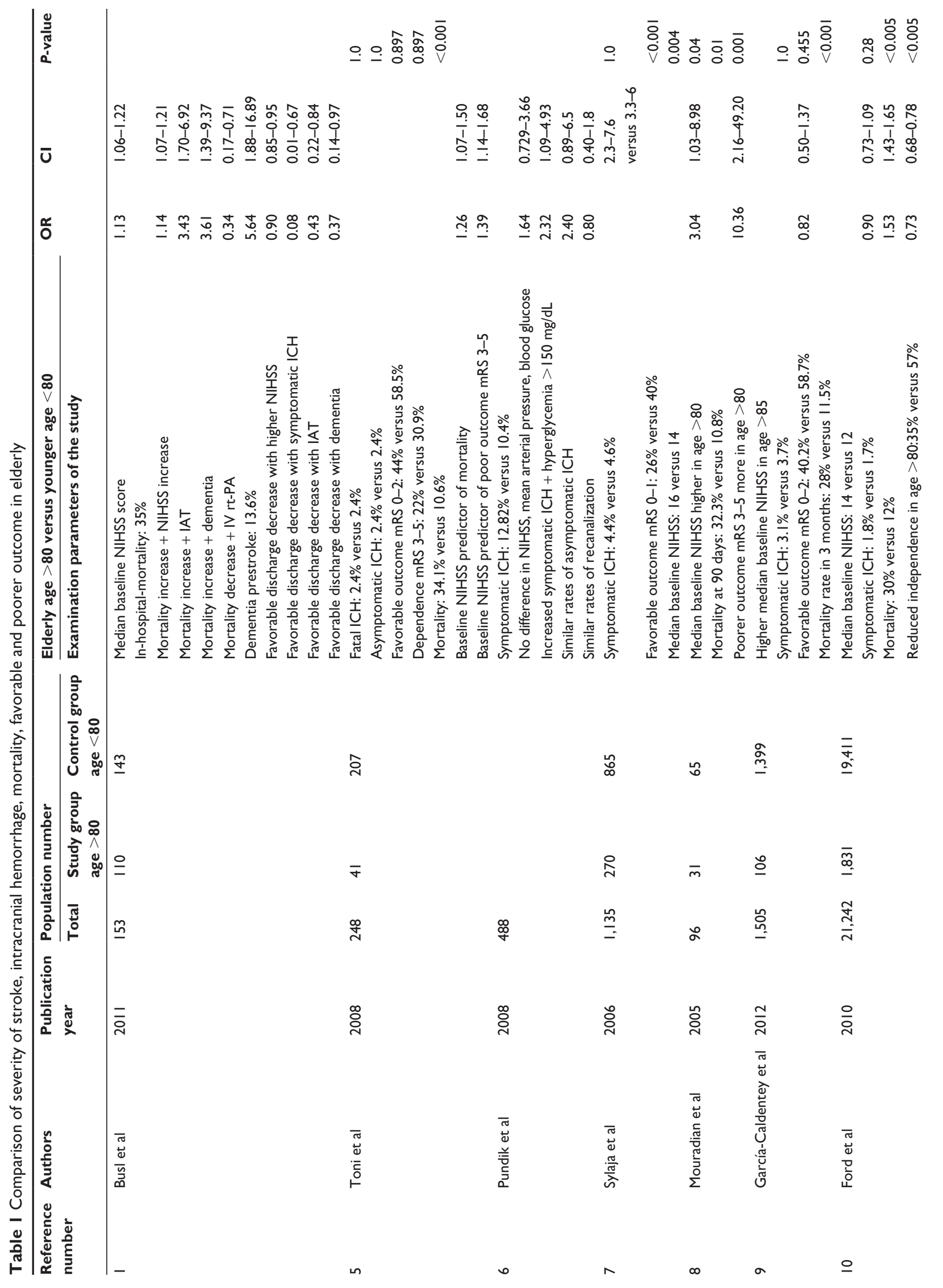




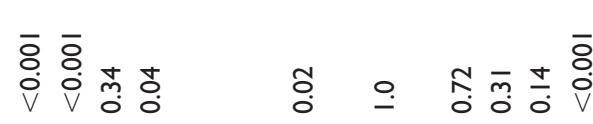

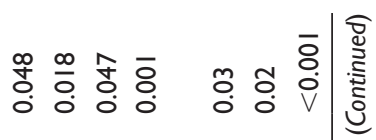

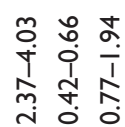

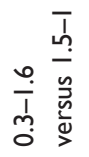

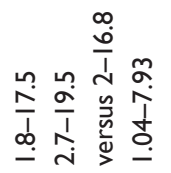

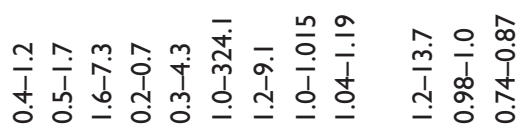

กุ้ กุ

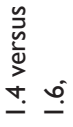

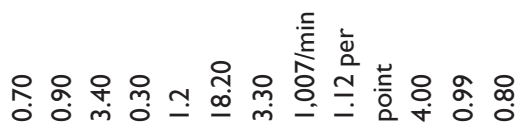

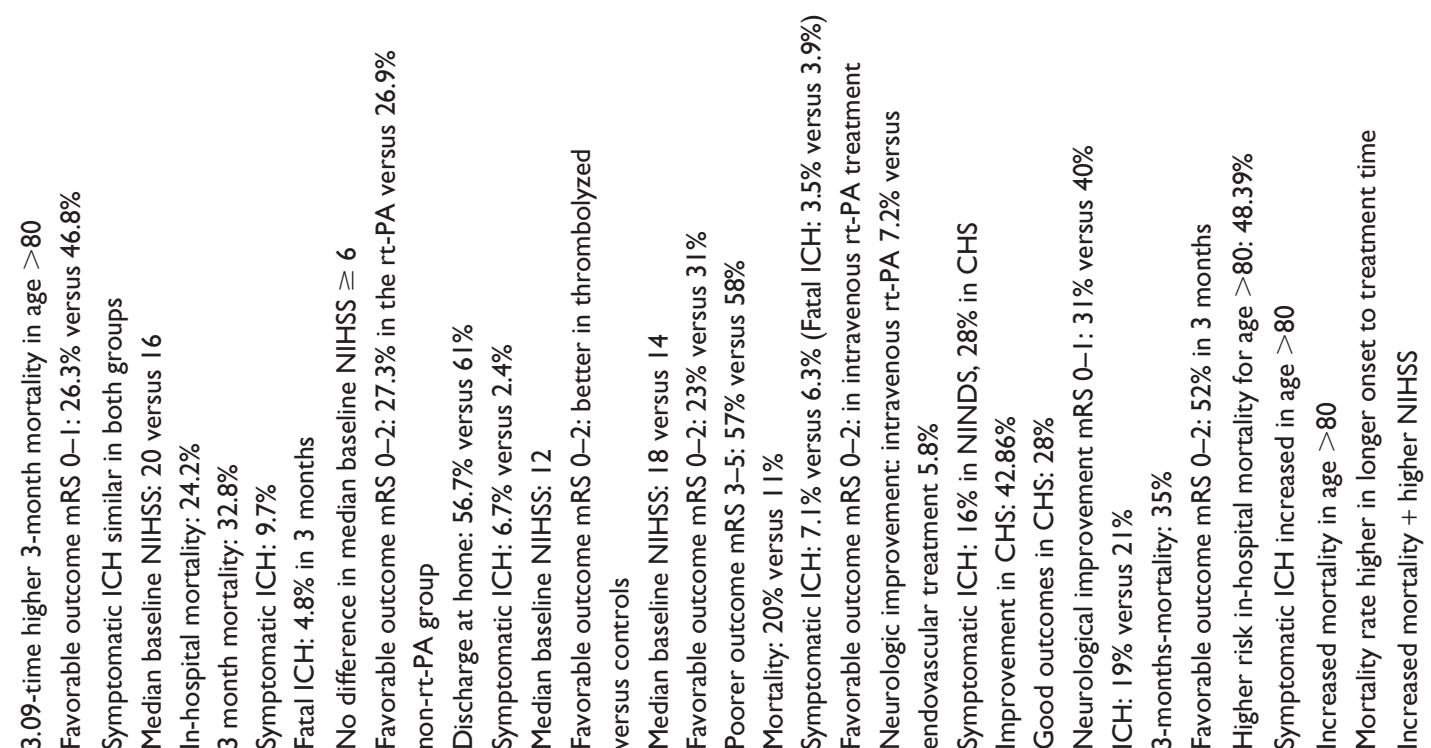

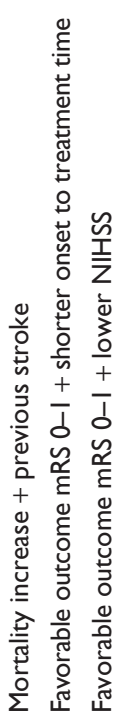

$\stackrel{5}{=}$

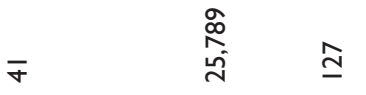

$\cong \stackrel{\text { స }}{=}$

$\bar{\infty} \cong$

ค

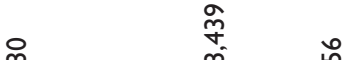

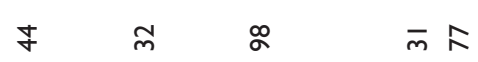

$\underset{4}{\stackrel{4}{*}}$

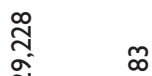

ํํำ 우용

ঃัن

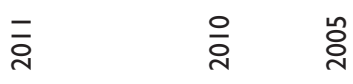

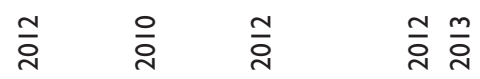

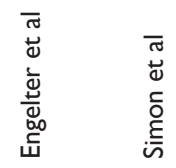

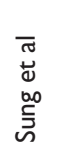

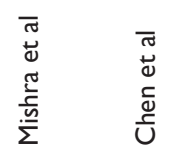

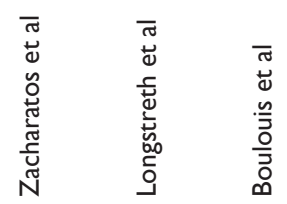

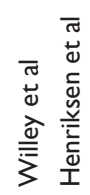

$=\quad \simeq$

$\underline{\underline{n}} \quad \underline{ \pm} \quad \underline{\underline{n}}$

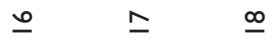

$\stackrel{4}{2}$ 


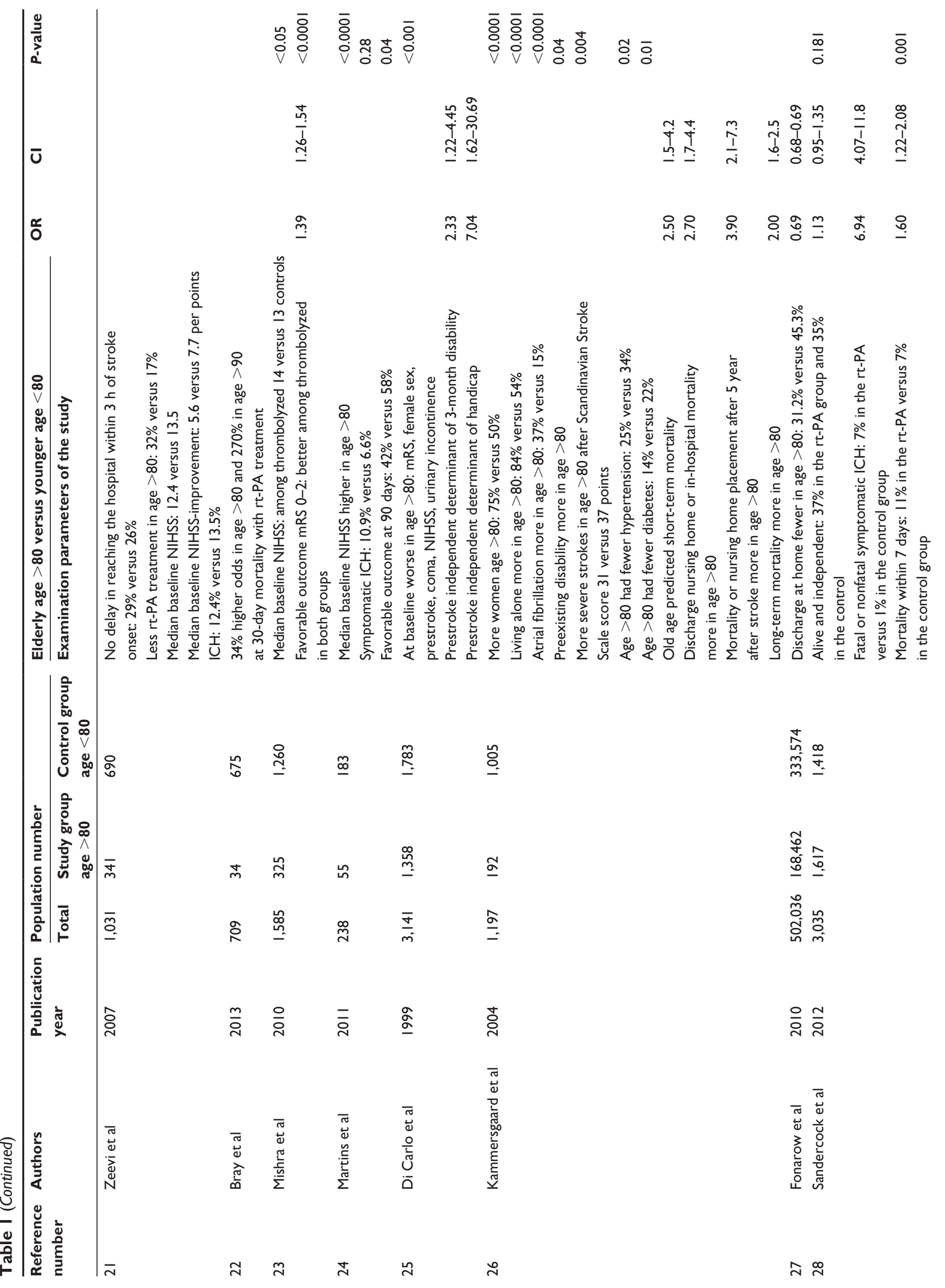




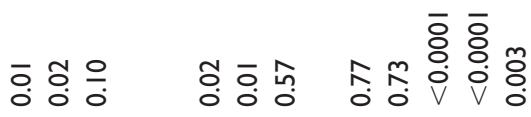

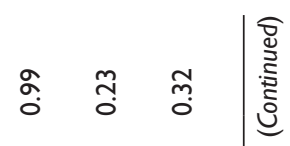

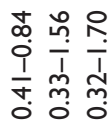

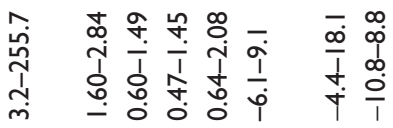

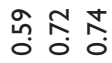

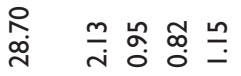

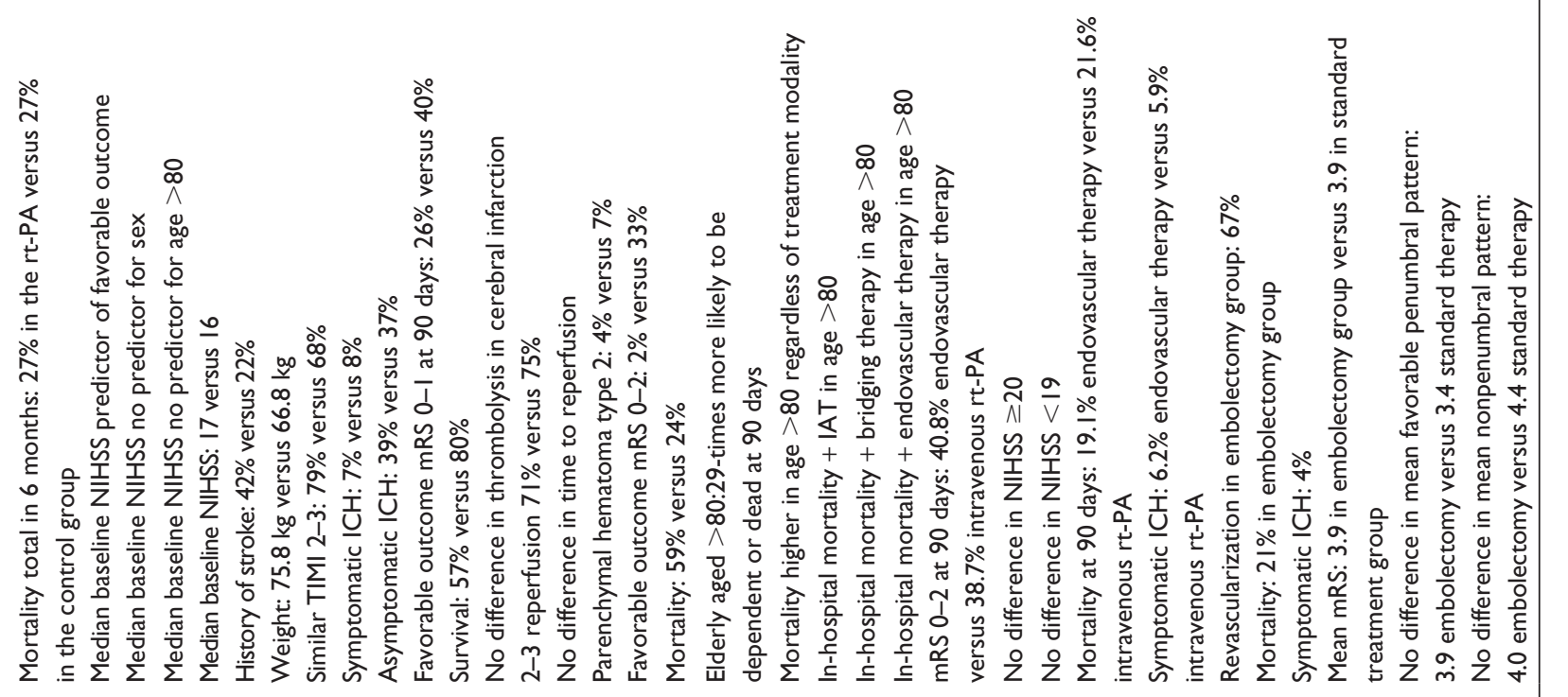

$\stackrel{\infty}{\circ} \bar{\infty} \quad \stackrel{\circ}{m} \stackrel{\stackrel{\infty}{\sim}}{\stackrel{\circ}{N}}$

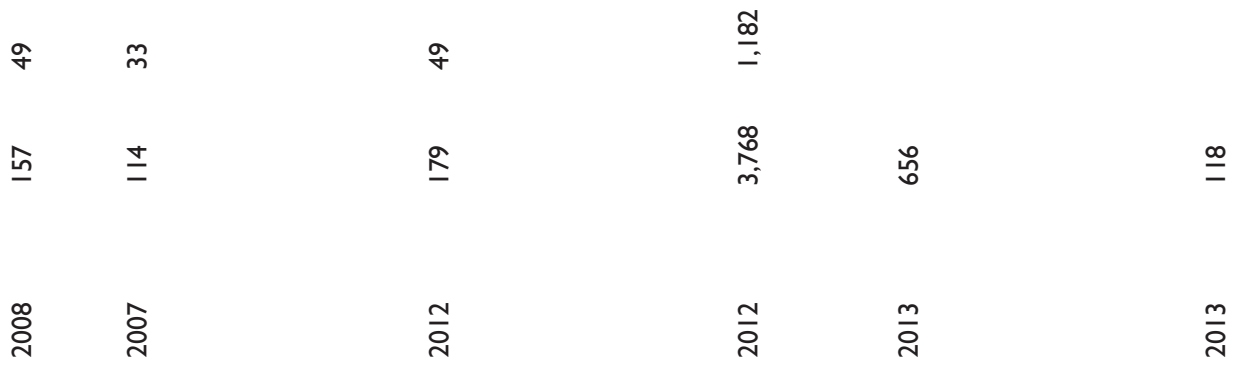

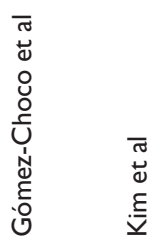

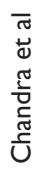

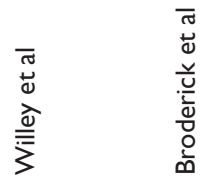

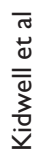

సे 이

$\bar{m}$

జี

m

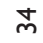


alteplase treatment protocol for unexplained reasons. These results, although requiring careful interpretation, suggest that alteplase treatment could be used in a community setting. ${ }^{21}$

Following a report by Bray et $\mathrm{a}^{22}$ in 2012, alteplase therapy has been more frequently administered to elderly stroke patients in England. Patients aged $>80$ years did not demonstrate an increased risk for severe complications following an acute ischemic stroke and alteplase treatment compared with younger patients, but the mortality rate was higher in the older group of patients. ${ }^{22}$

\section{Dementia}

Busl et $\mathrm{al}^{1}$ investigated dementia as a possible cause for refusing intravenous treatment with alteplase. In their study, all patients aged $>80$ years were enrolled after intravenous or intra-arterial thrombolytic therapy with rt-PA at a single institution. Data regarding existing cardiovascular risk factors and dementia were collected from the medical records of each patient. For this study, a patient was considered to have dementia if such a diagnosis was recorded in the medical history or the patient was receiving medical treatment for dementia. The primary study endpoint was in-hospital mortality, and the secondary endpoint was the destination following hospital discharge, eg, home or a rehabilitation facility. Destinations that included a nursing home, hospice, or death were considered unfavorable. Among the included patients, approximately more than two-thirds received intravenous alteplase, one-third received intra-arterial rt-PA, and a small number received both. The likelihood of death was associated with higher NIHSS scores, administration of intra-arterial reperfusion therapy, and the presence of dementia, but decreased with administration of intravenous rt-PA treatment. Increasing NIHSS scores and the presence of symptomatic intracranial hemorrhage, intra-arterial thrombolytic therapy, and dementia decreased the likelihood of a favorable discharge destination. In a multivariate analysis, only the NIHSS score and dementia independently predicted death and an unfavorable discharge destination (Table 1). Therefore, dementia seems to be a powerful independent predictor of in-hospital mortality following acute ischemic stroke, regardless of thrombolytic therapy. ${ }^{1}$

\section{Pretreatment severity}

According to the European Medicines Evaluation Agency, thrombolysis for acute ischemic stroke patients aged $>80$ years is not permitted in some countries due to limited safety and efficacy data. The effect of age on thrombolysis was examined in a neuroprotection study. Mishra et $\mathrm{al}^{23}$ 
compared patients who underwent and those who did not undergo thrombolysis as control regarding pretreatment severity (baseline NIHSS score), 90-day outcomes (mRS and NIHSS scores), and distribution of mRS scores, and used the Cochran-Mantel-Haenszel test and then the logistic regression, after adjusting for age and baseline NIHSS scores. According to the median baseline NIHSS scores, neurological symptoms were more severe in patients who underwent thrombolysis than in those who did not undergo thrombolysis; however, the mRS score distribution was better among patients who received thrombolytic therapy. This association occurred in younger and older patients (Table 1). The odds ratios were constant across all age deciles for more than 30 years, and the results, based on the NIHSS and dichotomized $\mathrm{mRS}$ scores, corroborated these findings. The outcomes for patients receiving thrombolysis treatment were significantly improved compared with those of control subjects. Despite the anticipated worse outcomes among the older patients compared to the younger patients, an association between thrombolysis and improved outcomes was observed in the very elderly. Therefore, age alone should not be a contraindication for thrombolysis therapy according to statements by the authors of this study. ${ }^{23}$

\section{Elderly patients in developing countries}

The mortality rate and burden after a stroke are high in developing countries due to many reasons. The number of stroke patients who receive rt-PA therapy is very low in developing countries. Financial problems, prehospital delay, and poor infrastructure are additional obstacles to thrombolytic therapy in developing countries. Therefore, elderly patients may be an important group in which new treatments for acute ischemic stroke should be considered, in particular in developing countries. A study investigated the use of alteplase in elderly Brazilian patients with acute ischemic stroke and compared the neurological presentation, imaging results, and occurrence of intracranial hemorrhages between those aged above and below 80 years of age, after treatment with alteplase. Higher prevalence of the female sex and a higher number of cases of previous transient ischemic attacks were observed in those aged $>80$ years. This age group also had elevated systolic blood pressures and higher NIHSS scores, whereas younger patients had higher serum glucose levels (Table 1). The rate of symptomatic cerebral hemorrhage was higher, but not statistically significant, in those aged $>80$ years. Among them, $6.6 \%$ achieved a positive outcome after 90 days, which was assessed after $\mathrm{mRS}$ score $\leq 1$, while a lower proportion of younger patients had the same outcomes. The outcomes were significantly worse among older patients diagnosed with an anterior circulation stroke, compared with younger patients with a similar diagnosis. Similarly, those aged $>80$ years had higher NIHSS scores, hypoattenuation in more than one third of the middle cerebral artery territory, and initial Alberta Stroke Program Early Computed Tomography scores of $\leq 7$. The results of this study support the use of alteplase in stroke patients aged $>80$ years who present early to hospitals in developing countries. ${ }^{24}$

\section{Age-related differences}

Previous studies have demonstrated a smaller benefit of alteplase in elderly patients, which may partially be due to increased stroke severity and higher preexisting stroke disability. ${ }^{25,26}$ Moreover, as expected, the outcome after stroke tends to be worse in elderly patients. Thus, age-related differences were examined among patients with acute ischemic stroke, regarding patient demographics, performance standards, time trends, and early clinical results in a large, contemporary cohort. The associations between age and clinical characteristics and outcomes noted in the hospital were analyzed in ischemic stroke patients from 1,256 hospitals in the Get With The Guidelines ${ }^{\circledR}$-Stroke program ${ }^{27}$ from 2003 to 2009; data were analyzed according to age groups and in 10-year periods. Seven preset ratios and two summary measures were also investigated in this study. Elderly patients were more likely to have preexisting atrial fibrillation or hypertension. This group included fewer Caucasians, Hispanics, and smokers; in addition, there were slight differences between the age groups. Improvements in treatment were seen in the performance standards from 2003 to 2009 in each age group, and many age-related treatment gaps were almost completely eliminated during this time period. Fewer elderly patients were discharged from the hospital to home, and in-hospital mortality increased for each 10-year age increase (Table 1). The neurological symptoms and mortality rates in the elderly patients were different from those in the younger patients. In acute ischemic stroke patients, performance-based treatments improved outcomes over time in all age groups, ultimately resulting in smaller age-related treatment gaps. ${ }^{27}$

The benefit of intravenous rt-PA administration in elderly aged $>80$ was demonstrated in another recent large study with more than half of the stroke patients aged $>80$ years. The results of this study showed no significant difference in the effect of treatment with rt-PA compared to the younger control groups aged $<80$ years. Elderly patients $>80$ years benefited more from treatment with rt-PA than those who did not receive rt-PA treatment in this trial. ${ }^{28}$ 


\section{Sex differences}

Sex differences in alteplase treatment for ischemic stroke were compared for elderly and younger patients in a recent study. ${ }^{29}$ The outcomes were NIHSS scores at 1 hour, 24 hours, and 7 days after rt-PA treatment. After 90 days, outcomes were also examined using the mRS, symptomatic cranial hemorrhage, and mortality. Logistic regression was applied to the baseline NIHSS scores, which were determined to be independent predictors of positive outcomes; the same association was not evident for sex or age $>80$ years (Table 1 ). This study also found that clinical improvement, mortality, and intracranial hemorrhage were not associated with age or sex in treatments involving alteplase. Therefore, age and sex were not observed to affect the efficacy of alteplase in treating acute ischemic stroke. $^{29}$

\section{Intra-arterial fibrinolysis}

Intra-arterial fibrinolytic therapy is an exciting off-label treatment for acute ischemic stroke. However, few data are available about this novel therapy in the elderly. One of the studies investigated the use of intra-arterial fibrinolysis therapy in elderly patients following acute ischemic stroke. Demographic data, complications, and outcomes were compared for an elderly group and a control group of younger counterparts over a study period of 9 years. Significant differences were not evident in patient demographics, and the patients demonstrated similar pretreatment NIHSS scores. The groups differed only in their past history of strokes or transient ischemic attacks and weight. Between the elderly and younger patients, no significant differences were found in the recanalization in thrombolysis in myocardial infarction rates, major symptomatic hemorrhages, or intracerebral hemorrhages. At 90 days, lower rates of good functional outcome and survival were noted in the elderly (Table 1). Thus, intra-arterial fibrinolysis can be performed in elderly patients with the same expected cerebral hemorrhage and recanalization rates as in younger patients. Although mortality rates were higher in the elderly group, and good functional outcomes were better in the younger control group, this study nevertheless argues for considering intra-arterial fibrinolysis in elderly patients. ${ }^{30}$

Intra-arterial therapy with rt-PA in patients was compared to intravenous treatment with rt-PA after acute ischemic stroke in patients aged $>80$ years and those aged $<80$ years. Patients aged $>80$ years had higher in-hospital mortality, regardless of the type of therapy administered. However, the intra-arterial therapy did not demonstrate an increased risk of in-hospital mortality compared with intravenous thrombolysis in the older patients. ${ }^{31}$

Different data exist for intra-arterial thrombolytic therapy in stroke patients aged $>80$ years. These elderly patients had worse outcomes after intra-arterial therapy with alteplase due to the severity of the stroke. Intra-arterial alteplase should be considered after careful evaluation of the risk and benefit in elderly patients. ${ }^{32}$

In addition, a recently published controlled trial showed no benefit of endovascular acute stroke therapy compared to rt-PA administered intravenously for patients with acute ischemic stroke. This study had to be prematurely interrupted because endovascular treatment did not yield any better results than the intravenous administration of rt-PA. ${ }^{33}$

According to the results in recent published reports, several trial outcomes showed that intravenous treatment with rt-PA was superior to intra-arterial fibrinolytic therapy and that intra-arterial treatment should be considered only in patients aged $>80$ years contraindicated for intravenous therapy with rt-PA. ${ }^{33,34}$

\section{Severe hemorrhagic complications}

Little clear information is available regarding the safety and efficacy of alteplase in treating acute ischemic stroke in patients aged $>80$ years. A study ${ }^{35}$ examined the outcomes and severe hemorrhagic complications in this population using data from three German stroke centers. The stroke severity was evaluated using the NIHSS scores, and a positive outcome after alteplase was defined as a mRS score of $0-1$. Patients who experienced severe intracerebral bleeding as a complication of alteplase underwent follow-up magnetic resonance imaging or cranial computed tomography. Of the patients who received intravenous alteplase for an acute ischemic stroke, $16 \%$ were aged $>80$ years. There were no significant differences in the NIHSS scores upon admission or in the onset of alteplase treatment time between the younger and older patients. The elderly patients had fewer positive outcomes compared to the younger group and had a higher mortality rate. However, significant differences were not observed in the parenchymal hemorrhage and symptomatic intracerebral hemorrhage rates for these groups (Table 1). Despite similar intracranial bleeding rates, the elderly patients experienced worse outcomes. Nevertheless, no evidence demonstrated more complications due to alteplase in elderly patients aged $>80$ years. Therefore, this study suggests that age should not be an exclusion criterion for alteplase treatment for acute ischemic stroke. ${ }^{35}$ 


\section{Efficacy of alteplase in patients over 90 years of age}

Although the risk of stroke increases with age, some physicians remain cautious about administering thrombolytic therapy to very elderly patients who experience an acute ischemic stroke. Mateen et $\mathrm{al}^{36}$ were the first to examine alteplase treatment in patients aged $>90$ years, using retrospective data from four medical centers between 1999 and 2008. The authors examined clinical neurological symptoms, complications, and outcomes of patients with acute ischemic stroke after alteplase treatment. The study included eleven women, with age ranging from 90-101 years. The range of time to alteplase treatment was $90-180$ minutes. The patients had a range of NIHSS scores of 5-28. Nearly all patients were mobile before the stroke (median mRS score, 1 ; median Barthel Index score, 95); only one patient had minimal movement restrictions. After 30 days of observation, two patients had a positive outcome, and two others had slight disabilities. The majority of patients died or had severe mobility problems, and three had asymptomatic cerebral hemorrhages (Table 1). Thus, alteplase treatment did not appear to improve outcomes. ${ }^{36}$ However, better results were obtained with alteplase treatment in patients with acute ischemic stroke aged $>90$ years in another recently published study. ${ }^{28}$ In this study, 111 patients with acute ischemic stroke aged $>90$ years were treated with rt-PA. The majority of patients aged $>90$ years benefited from treatment with rt-PA within the 3-hour treatment time frame in this study. ${ }^{28}$

\section{Conclusion}

The proportion of elderly patients experiencing strokes is approximately $30 \%$. Nonetheless, they receive suboptimal acute therapy and management. Elderly patients should be treated in a stroke unit instead of a general ward, and early rehabilitation is warranted for these patients. Aspirin should be administered to elderly stroke victims within 48 hours of symptom onset to reduce recurrence and mortality. Age alone should not be considered a contraindication for administering intravenous alteplase. Instead, the benefits and risks should be weighed to determine the best course of treatment for the individual patient. In addition, coordinated clinical procedures will help ensure the care of elderly stroke patients. ${ }^{37}$

Advanced age should not be a contraindication for alteplase treatment in patients who have suffered an acute ischemic stroke. Instead, the attending physician should decide on the course of treatment on a case-by-case basis, carefully weighing the risks and benefits of thrombolytic therapy for each patient. For example, dementia can affect the outcome following alteplase treatment. The incidence of adverse effects such as intracranial bleeding as well as the severity of acute cerebral ischemia may increase with age. However, some studies have shown that serious complications such as heavy intracranial bleeding due to alteplase were not significantly increased in elderly patients. The use of alteplase in elderly acute ischemic stroke patients can also be applied in developing countries. Furthermore, there is no evidence of sex- or age-related complications associated with the use of alteplase. In patients aged $>90$ years, alteplase treatment did not improve outcomes relative to untreated patients. However, intra-arterial fibrinolysis should be considered only in extremely carefully selected patients aged $>80$ years, since the benefits are not clear even in younger patients. As poststroke life expectancies have improved in recent decades, alteplase should not be categorically denied to elderly patients with acute ischemic stroke, based solely on patient age. Because the threshold of 80 years is arbitrary for thrombolysis, physicians should weigh the risks and benefits of intravenous alteplase to treat acute ischemic stroke in elderly patients on an individual basis.

\section{Disclosure}

The author reports no conflicts of interest in this work.

\section{References}

1. Busl KM, Nogueira RG, Yoo AJ, Hirsch JA, Schwamm LH, Rost NS Prestroke dementia is associated with poor outcomes after reperfusion therapy among elderly stroke patients. J Stroke Cerebrovasc Dis. Epub December 17, 2011

2. Tanne D, Turgeman D, Adler Y. Management of acute ischaemic stroke in the elderly: tolerability of thrombolytics. Drugs. 2001;61(10): 1439-1453.

3. Del Zoppo GJ, Saver JL, Jauch EC, Adams HP Jr; American Heart Association Stroke Council. Expansion of the time window for treatment of acute ischemic stroke with intravenous tissue plasminogen activator: a science advisory from the American Heart Association/American Stroke Association. Stroke. 2009;40(8):2945-2948.

4. Lorenzano S, Toni D; TESPI trial Investigators. TESPI (Thrombolysis in Elderly Stroke Patients in Italy): a randomized controlled trial of alteplase (rt-PA) versus standard treatment in acute ischaemic stroke in patients aged more than 80 years where thrombolysis is initiated within three hours after stroke onset. Int J Stroke. 2012;7(3):250-257.

5. Toni D, Lorenzano S, Agnelli G, et al. Intravenous thrombolysis with rt-PA in acute ischemic stroke patients aged older than 80 years in Italy. Cerebrovasc Dis. 2008;25(1-2):129-135.

6. Pundik S, McWilliams-Dunnigan L, Blackham KL, et al. Older age does not increase risk of hemorrhagic complications after intravenous and/or intra-arterial thrombolysis for acute stroke. J Stroke Cerebrovasc Dis. 2008;17(5):266-272.

7. Sylaja PN, Cote R, Buchan AM, Hill MD; Canadian Alteplase for Stroke Effectiveness Study (CASES) Investigators. Thrombolysis in patients older than 80 years with acute ischaemic stroke: Canadian Alteplase for Stroke Effectiveness Study. J Neurol Neurosurg Psychiatr. 2006;77(7):826-829 
8. Mouradian MS, Senthilselvan A, Jickling G, et al. Intravenous rt-PA for acute stroke: comparing its effectiveness in younger and older patients. J Neurol Neurosurg Psychiatr. 2005;76(9):1234-1237.

9. García-Caldentey J, Alonso de Leciñana M, Simal P, et al. Intravenous thrombolytic treatment in the oldest old. Stroke Res Treat. 2012;2012: 923676.

10. Ford GA, Ahmed N, Azevedo E, et al. Intravenous alteplase for stroke in those older than 80 years old. Stroke. 2010;41(11):2568-2574.

11. Engelter ST, Bonati LH, Lyrer PA. Intravenous thrombolysis in stroke patients of $\geq 80$ versus $<80$ years of age - a systematic review across cohort studies. Age Ageing. 2006;35(6):572-580.

12. Simon JE, Sandler DL, Pexman JH, Hill MD, Buchan AM; Calgary Stroke Programme. Is intravenous recombinant tissue plasminogen activator (rt-PA) safe for use in patients over 80 years old with acute ischaemic stroke? - The Calgary experience. Age Ageing. 2004;33(2):143-149.

13. Sung PS, Chen $\mathrm{CH}$, Hsieh $\mathrm{HC}$, et al. Outcome of acute ischemic stroke in very elderly patients: is intravenous thrombolysis beneficial? Eur Neurol. 2011;66(2):110-116.

14. Mishra NK, Ahmed N, Andersen G, et al; SITS collaborators. Thrombolysis in very elderly people: controlled comparison of SITS International Stroke Thrombolysis Registry and Virtual International Stroke Trials Archive. BMJ. 2010;341:c6046.

15. Chen CI, Iguchi Y, Grotta JC, et al. Intravenous TPA for very old stroke patients. Eur Neurol. 2005;54(3):140-144.

16. Zacharatos H, Hassan AE, Vazquez G, et al. Comparison of acute nonthrombolytic and thrombolytic treatments in ischemic stroke patients 80 years or older. Am J Emerg Med. 2012;30(1):158-164.

17. Longstreth WT Jr, Katz R, Tirschwell DL, Cushman M, Psaty BM. Intravenous tissue plasminogen activator and stroke in the elderly. Am J Emerg Med. 2010;28(3):359-363.

18. Boulouis G, Dumont F, Cordonnier C, Bodenant M, Leys D, Hénon H. Intravenous thrombolysis for acute cerebral ischaemia in old stroke patients $\geq 80$ years of age. J Neurol. 2012;259(7):1461-1467.

19. Willey JZ, Petersen N, Dhamoon MS, et al. Safety of thrombolysis in patients over the age of 80. Neurologist. 2012;18(2):99-101.

20. Henriksen EH, Ljøstad U, Tveiten A, Naess H, Thomassen L, Mygland A. TPA for ischemic stroke in patients $\geq 80$ years. Acta Neurol Scand. 2013;127(5):309-315.

21. Zeevi N, Chhabra J, Silverman IE, Lee NS, McCullough LD. Acute stroke management in the elderly. Cerebrovasc Dis. 2007;23(4):304-308.

22. Bray BD, Campbell J, Hoffman A, Tyrrell PJ, Wolfe CD, Rudd AG. Stroke thrombolysis in England: an age stratified analysis of practice and outcome. Age Ageing. 2013;42(2):240-245.

23. Mishra NK, Diener HC, Lyden PD, Bluhmki E, Lees KR; VISTA Collaborators. Influence of age on outcome from thrombolysis in acute stroke: a controlled comparison in patients from the Virtual International Stroke Trials Archive (VISTA). Stroke. 2010;41(12):2840-2848.
24. Martins SC, Friedrich MA, Brondani R, et al. Thrombolytic therapy for acute stroke in the elderly: an emergent condition in developing countries. J Stroke Cerebrovasc Dis. 2011;20(5):459-464.

25. Di Carlo A, Lamassa M, Pracucci G, et al. Stroke in the very old: clinical presentation and determinants of 3-month functional outcome: A European perspective. European BIOMED Study of Stroke Care Group. Stroke. 1999;30(11):2313-2319.

26. Kammersgaard LP, Jørgensen HS, Reith J, et al; Copenhagen Stroke Study. Short- and long-term prognosis for very old stroke patients. The Copenhagen Stroke Study. Age Ageing. 2004;33(2):149-154.

27. Fonarow GC, Reeves MJ, Zhao X, et al; Get With the Guidelines-Stroke Steering Committee and Investigators. Age-related differences in characteristics, performance measures, treatment trends, and outcomes in patients with ischemic stroke. Circulation. 2010;121(7):879-891.

28. Sandercock P, Wardlaw JM, Linley RI, et al; IST-3 collaborative group. The benefits and harms of intravenous thrombolysis with recombinant tissue plasminogen activator within $6 \mathrm{~h}$ of acute ischaemic stroke (the third international stroke trial [IST-3]): a randomised controlled trial. Lancet. 2012;379(9834):2352-2363.

29. Gómez-Choco M, Obach V, Urra X, et al. The response to IV rt-PA in very old stroke patients. Eur J Neurol. 2008;15(3):253-256.

30. Kim D, Ford GA, Kidwell CS, et al; UCLA Intra-Arterial Thrombolysis Investigators. Intra-arterial thrombolysis for acute stroke in patients 80 and older: a comparison of results in patients younger than 80 years. AJNR Am J Neuroradiol. 2007;28(1):159-163.

31. Chandra RV, Leslie-Mazwi TM, Oh DC, et al. Elderly patients are at higher risk for poor outcomes after intra-arterial therapy. Stroke. 2012;43(9):2356-2361.

32. Willey JZ, Ortega-Gutierrez S, Petersen N, et al. Impact of acute ischemic stroke treatment in patients $>80$ years of age: the specialized program of translational research in acute stroke (SPOTRIAS) consortium experience. Stroke. 2012;43(9):2369-2375.

33. Broderick JP, Palesch YY, Demchuk AM, et al; Interventional Management of Stroke (IMS) III Investigators. Endovascular therapy after intravenous t-PA versus t-PA alone for stroke. $N$ Engl J Med. 2013;368(10):893-903.

34. Kidwell CS, Jahan R, Gornbein J, et al; MR RESCUE Investigators. A trial of imaging selection and endovascular treatment for ischemic stroke. N Engl J Med. 2013;368(10):914-923.

35. Berrouschot J, Röther J, Glahn J, Kucinski T, Fiehler J, Thomalla G. Outcome and severe hemorrhagic complications of intravenous thrombolysis with tissue plasminogen activator in very old ( $\geq 80$ years) stroke patients. Stroke. 2005;36(11):2421-2425.

36. Mateen FJ, Nasser M, Spencer BR, et al. Outcomes of intravenous tissue plasminogen activator for acute ischemic stroke in patients aged 90 years or older. Mayo Clin Proc. 2009;84(4):334-338.

37. Derex L. Acute stroke management in elderly patients. Rev Prat. 2012;62(9):1225-1228. French.
Clinical Interventions in Aging

\section{Publish your work in this journal}

Clinical Interventions in Aging is an international, peer-reviewed journal focusing on evidence-based reports on the value or lack thereof of treatments intended to prevent or delay the onset of maladaptive correlates of aging in human beings. This journal is indexed on PubMed Central, MedLine, the American Chemical Society's 'Chemical Abstracts

\section{Dovepress}

Service' (CAS), Scopus and the Elsevier Bibliographic databases. The manuscript management system is completely online and includes a very quick and fair peer-review system, which is all easy to use. Visit $\mathrm{http}: / /$ www.dovepress.com/testimonials.php to read real quotes from published authors. 